\title{
Deep brain stimulation: a return journey from psychiatry to neurology
}

\author{
Keyoumars Ashkan, ${ }^{1}$ Paul Shotbolt, ${ }^{2}$ Anthony S David, ${ }_{1}^{3}$ Michael Samuel ${ }^{4}$
}

${ }^{1}$ Department of Neurosurgery, King's College Hospital NHS

Foundation Trust, King's Health Partners, London, UK

${ }^{2}$ Neuropsychiatry Service, South London and The

Maudsley NHS Foundation Trust, King's Health Partners, London, UK

${ }^{3}$ Department of Cognitive Neuropsychiatry, Institute of Psychiatry, King's Health Partners, London, UK ${ }^{4}$ Department of Neurology, King's College Hospital NHS Foundation Trust, King's Health Partners, London, UK

\section{Correspondence to}

Keyoumars Ashkan,

Department of Neurosurgery, King's College Hospital, Denmark Hill, London SE5 9RS, UK; k.ashkan@nhs.net

Received 5 October 2012 Revised 7 January 2013 Accepted 24 February 2013 Published Online First 15 March 2013

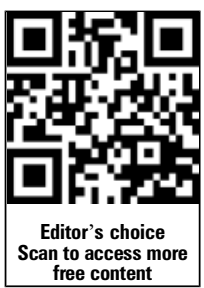

To cite: Ashkan $\mathrm{K}$ Shotbolt P, David AS, et al. Postgrad Med J

2013;89:323-328

\section{ABSTRACT}

Deep brain stimulation (DBS) has emerged as an effective neurosurgical tool to treat a range of conditions. Its use in movement disorders such as Parkinson's disease, tremor and dystonia is now well established and has been approved by the National Institute of Clinical Excellence (NICE). The NICE does, however, emphasise the need for a multidisciplinary team to manage these patients. Such a team is traditionally composed of neurologists, neurosurgeons and neuropsychologists. Neuropsychiatrists, however, are increasingly recognised as essential members given many psychiatric considerations that may arise in patients undergoing DBS. Patient selection, assessment of competence to consent and treatment of postoperative psychiatric disease are just a few areas where neuropsychiatric input is invaluable. Partly driven by this close team working and partly based on the early history of DBS for psychiatric disorders, there is increasing interest in re-exploring the potential of neurosurgery to treat patients with psychiatric disease, such as depression and obsessive-compulsive disorder. Although the clinical experience and evidence with DBS in this group of patients are steadily increasing, many questions remain unanswered. Yet, the characteristics of optimal surgical candidates, the best choice of DBS target, the most effective stimulating parameters and the extent of postoperative improvement are not clear for most psychiatric conditions. Further research is therefore required to define how DBS can be best utilised to improve the quality of life of patients with psychiatric disease.

\section{INTRODUCTION}

Cerebral neurological and psychiatric disorders have been divided into different medical specialties in some countries, whereas in others they are broadly managed together. Clear distinctions between organic neuropsychiatry and psychiatric or psychological aspects of neurological disorders are often imprecise, but these disorders provide neurologists and psychiatrists with opportunities to combine expertise for optimal patient care and research. The recent expansion of deep brain stimulation (DBS) in the treatment of both neurological and psychiatric disorders has re-introduced neurosurgeons to these multidisciplinary teams. DBS is a powerful neurosurgical technique that allows modulation of brain physiology. As we better understand the abnormal circuitry underpinning brain dysfunction in different disease states, DBS may well be increasingly called upon as a means of altering the pathological circuits to near physiological states.

In this article, we first review the current practice of DBS primarily to treat neurological movement disorders. We will then move on to highlight the psychiatric considerations that may arise in treating these patients. Finally, we will discuss the future direction of DBS, particularly its potential to treat primary psychiatric disorders. We hope this review demonstrates how the recent advances in DBS have permitted its use in the treatment of neurological movement disorders and psychiatric diseases, both separately and also at their interface, for the benefit of both.

\section{BACKGROUND AND CURRENT INDICATIONS IN NEUROLOGY}

DBS is a treatment strategy, which involves delivering targeted electrical stimulation to specific brain regions, using permanently placed small electrodes, for the alleviation of symptoms. The initial development and indeed application of the technique in 1940s and 1950s were for patients with psychiatric disease, ${ }^{1}$ largely driven by the need for safer surgical strategies than were available (eg, lobotomy) at the time. ${ }^{2}$ Subsequent developments in psychopharmacology and other psychiatric treatment strategies, however, minimised the need for surgical interventions for psychiatric symptoms, essentially leading to its abandonment.

The widespread resurgence of DBS into mainstream medicine in the recent decades is largely owed to the pioneering work of Benabid and colleagues ${ }^{34}$ in the field of movement disorders. Before its introduction, movement disorders, such as refractory tremor or dystonia, could be improved by the stereotactic placement of small lesions in well-defined motor nuclei (eg, ventral thalamotomy). Lesional therapy, however, has three main drawbacks: lack of reversibility, lack of ability to adjust a lesion and an unacceptably high side-effect profile when bilateral lesions are performed. ${ }^{5}$ DBS offered arguably equivalent therapeutic benefit, but with the ability to tailor stimulation parameters (amplitude, frequency and pulse width of the current delivered) to patients' responses. In addition, innovative electrode design with four active contacts at the tip (figure 1) means that the DBS current can be anatomically varied if required over the active length of the electrode, increasing its versatility to move current to target structures (to increase clinical benefit) or move current away from unwanted structures (should electrical stimulation-induced side effects occur).

To place the DBS electrode, the neurosurgery relies on stereotactic techniques, essentially using high-quality MRI images to identify the deep brain targets and calculate their coordinates with respect to fixed landmarks of the stereotactic frame. ${ }^{6}$ For those targets not visible on MRI scans, coordinates are derived from standard brain atlases. The 


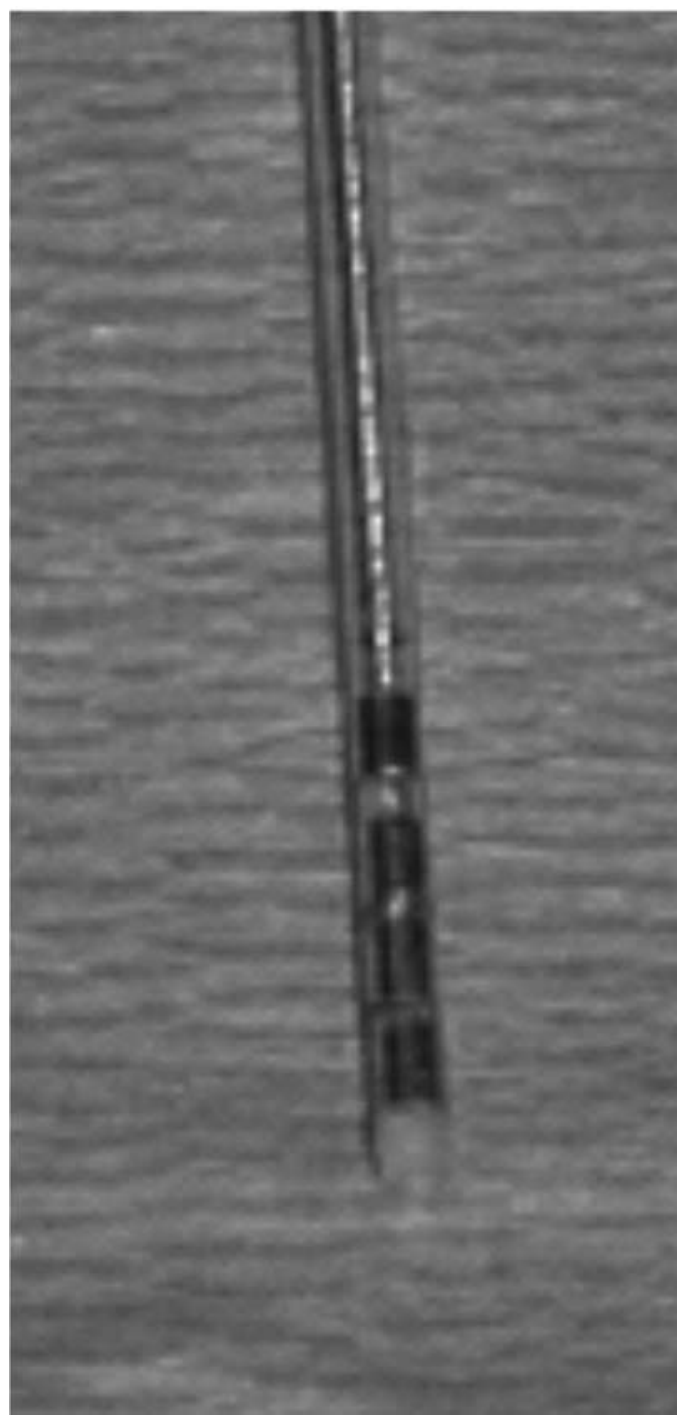

Figure 1 Deep brain stimulation electrode with the four active (metallic) contacts through which electricity is delivered to the brain tissue.

electrode is then passed to the anatomical target through a small burr hole made in the skull. Given the usually small size of the targets, their complex physiology and also proximity to other vital structures, most centres complement the anatomical targeting with intraoperative physiological evaluation to locate the optimal location within the anatomical target area. These may include clinical test stimulation during intraoperative evaluation in an awake patient operated under local anaesthesia or various forms of intraoperative electrophysiological recordings. Once the electrode is in position, it is connected to a battery pacemaker, placed in a subcutaneous pocket usually below the clavicle, to generate stimulation. In the vast majority of the patients, the operation is performed bilaterally; although occasionally in unilateral disease, a single electrode may suffice. The system is fully externally programmable and there is now a choice of fixed life or rechargeable batteries available.

For movement disorders, its most frequent clinical application has been for Parkinson's disease (PD). Typically in PD, symptoms of bradykinesia, tremor and rigidity respond very well to medical therapy with levodopa preparations or dopamine agonists over the first few years of the illness. With disease progression, a small but significant proportion of patients, however, develop late complications of medical therapy, which can become very disabling. Although the response to medication persists, it can become unpredictable, leading to on-off motor fluctuations and dyskinesia which are problematic when 'on'. The underlying parkinsonism remains problematic when 'off'; so the patient experiences impairment of activity of daily living. Tremor may also remain resistant to high-dose medication. Under these circumstances, three large controlled trials of DBS in PD have shown significant and concordant effects of DBS at providing an approximately $37 \%-54 \%$ reduction of complications of therapy or dyskinesia, allowing an overall reduction of antiparkinsonian medication by approximately 23\%-50\% and leading to an improvement in the overall quality of life of $14 \%$ $24 \%$, compared with best medical therapy. ${ }^{7-9}$ Trials are, however, only 6-12 months in duration, although non-trial data support the sustainable effect of DBS for up to 10 years in wellselected cases. These PD patients mostly had DBS of the subthalamic nucleus. For primary and secondary dystonia, the target is the internal segment of the globus pallidus, ${ }^{10}$ whereas for essential tremor, the target is usually the ventral thalamus. ${ }^{11}$ In the UK, DBS is approved by the National Institute of Clinical Excellence (NICE) for the management of these disorders, with patient selection and postoperative surveillance by a multidisciplinary team (MDT) being a central requirement. ${ }^{12} 13$

The clinical use of DBS for movement disorders was based on and is well supported by experimental data of abnormal neuronal firing patterns at these target nuclei. ${ }^{14}$ This, in conjunction with the recognition of the existence of the basal ganglia-thalamocortical circuits, ${ }^{15}$ offers scientific models of how DBS provides its clinical effect. Since its clinical effect is similar to that of a lesion, it has been perceived that DBS acts by producing a lesion-like effect at the target site. It is likely, however, that this is too simplistic. Advanced human intraoperative electrophysiological recordings in patients with PD have suggested that the dopamine-depleted 'motor' circuit may have an abnormal oscillation at about $\beta$ frequencies. ${ }^{16}$ One hypothesis of the mode of action of DBS is that DBS releases the 'motor' circuit from this abnormal oscillation, thus permitting a movement to occur without the influence of the pathological oscillation. Electrical stimulation at other anatomical structures within a circuit could be hypothesized to reduce abnormal oscillation within a circuit. However, for movement disorders, stimulation of other structures has not been performed with significant success and the subcortical structures remain the sites of choice for utmost efficacy with least motor side effect. The expansion of DBS for psychiatric indications, however, has not been limited to subcortical structures.

\section{PSYCHIATRIC CONSIDERATIONS WHEN SELECTING PATIENTS FOR DBS}

Patient selection for DBS for movement disorders, such as PD, is based on generally agreed guidelines. ${ }^{17}$ These include medically refractory symptoms and or signs and an absence of untreated general medical, major cognitive or psychiatric symptoms.

The latter is of particular importance as DBS, specifically of the subthalamic nucleus, is reported to have psychiatric complications that may include transient aggression, hypomania, mania, depression, anxiety, apathy and suicide. ${ }^{18}$ An overall suboptimal outcome may be related to a range of psychiatric, social, neurological and surgical variables. ${ }^{19}$ Cognitive problems have also been reported. The most robust finding is a decline in verbal fluency, even though declines in verbal memory, visuospatial memory and executive function have also been reported. ${ }^{20}$ 
To identify the patients at risk, treat the risk factors before proceeding to surgery or not to proceed if the risk factors cannot be managed, all patients being considered for DBS should undergo comprehensive neuropsychiatric and neuropsychological assessment. Patients attending DBS clinics for consideration of surgery have high rates of current and past psychopathology. ${ }^{21}$ Psychiatric disorders may increase the risk of poor outcome following surgery or affect patients' assessment of their difficulties, or even their capacity to consent to surgical intervention. At our centre, we have implemented a 'one-stop' DBS clinic in which the patient can see the neurologist, neurosurgeon and neuropsychiatrist sequentially in the same clinic location. This has dramatically streamlined the DBS care pathway for the movement disorder patients, and has obviated the need for multiple visits to different parts of the hospital.

The neuropsychiatric assessment consists of a clinical interview, informant interview and mental state examination. Rating scales such as the Beck Depression Inventory and the Hospital Anxiety and Depression Scale can be used to provide more quantitative measures useful for preoperative and postoperative comparisons. $^{22}{ }^{23}$ Formal neuropsychometric testing, taking a longer time, is performed separately by a neuropscyhologist and includes tests for intellect, executive, language, memory and perceptual functions. Rating scales assessing psychiatric and psychological symptoms complement clinical evaluation and, at present, cannot be used reliably to provide clinical cut-offs to distinguish between good and poor candidates. The decision must be based on the overall clinical picture and is made by a multidisciplinary team comprising neurologists, neurosurgeons, neuropsychiatrists, neuropsychologists and specialist nurses.

Particular attention is paid to current or historical psychotic symptoms and their relationship to medication. Patients with current active psychosis require treatment prior to having surgery. This may mean adjustment of antiparkinsonian medication or addition of antipsychotic drugs. Current depression also requires treatment with antidepressants or psychotherapy if necessary. In the case of depression, particularly, given its high prevalence, it would be wrong to refuse treatment on the basis of past disorder. Indeed, many patients with PD are highly motivated to receive DBS and it would be regrettable, not to mention dangerous, if they felt it necessary to conceal a psychiatric history for fear that it would preclude them from the intervention. A history of psychosis or depression does dictate that postoperative monitoring for recurrence of these symptoms is essential.

With regard to the impulse control disorders, such as pathological gambling and hypersexuality, there remains some debate on the preoperative management and postoperative course when these conditions co-exist with intractable motor signs in PD. There are reports of both worsening ${ }^{18} 2425$ and improvement postoperatively. ${ }^{26} 27$

In patients in whom impulse control disorders are secondary to dopaminergic medications, it has been suggested that the postoperative dopaminergic drug reduction may help in improving the impulse control disorders. However, it has also been suggested that impulse control disorders can develop post-DBS as a direct result of stimulation. ${ }^{28}$ Preoperative screening and treatment for these conditions are strongly recommended. It is important to remember that patients may not report these disorders, even on direct questioning. A collaborative history from carers or relatives is therefore crucial.

Symptoms of obsessive-compulsive disorder (OCD) are again prevalent in the cohort being assessed for DBS, particularly in PD patients. ${ }^{29}$ There are reports of improvements in OCD symptoms post-DBS, ${ }^{30} 31$ and indeed subthalamic nucleus (STN)-DBS has been investigated as a treatment for OCD in its own right. $^{32}$ Nevertheless, conventional treatment of OCD symptoms, usually with combined pharmacological and expert cognitive-behavioural therapies, is recommended before progressing to DBS.

Cognitive functioning can be assessed by the psychiatrist using the Addenbrooke's Cognitive Examination ${ }^{33}$ and more formally by the neuropsychologist of the team. The presence of a moderate-to-severe dementing illness is a contraindication to surgery for two reasons: first, because the procedure can have deleterious effects on cognition ${ }^{20}$ and second because the gains in quality of life for the patient post-DBS are less apparent.

A further important role of the neuropsychiatrist is to advise in the assessment of patient's capacity to give consent for the procedure. As the Mental Capacity Act instructs, the patient must be able to understand, retain and weigh up the information presented to them about the procedure. ${ }^{34}$ As such, it is important that they are cognisant of the risks of the operation. These include around $1 \%$ risk of major morbidity or mortality due to haemorrhage, stroke or seizures, $2 \%-10 \%$ risk of infection and a 5\%-10\% risk of changes in mood, cognition and behaviour. It is also important to ascertain the patient's beliefs about the illness, the procedure and the prognosis post surgery.

Detailed information on patients' comprehension of DBS surgery is lacking. Although it is intuitive that patients can understand some types of risks, such as infection, it is unclear as to what extent patients and carers, who have, for example, never experienced apathy or depression, can appreciate the meaning and social implication of these should they occur postoperatively. A proportion of patients undergoing assessment have unrealistic expectations about DBS. Such unrealistic expectations need to be identified and subsequently discussed and addressed by the DBS MDT. In this process, it is important to involve the patient's carers. The carers also need to be educated about the risks and benefits of the procedure to enable them to provide support throughout the process.

Further research is required before definitive statements can be made about which psychiatric conditions in patients with neurological disease may be absolute or relative contraindications to surgery. Follow-up of patients will inform us about the possible psychiatric risk factors and in turn allow us to give our patients more comprehensive information about the possible psychiatric adverse effects.

\section{PSYCHIATRIC INDICATIONS AND FUTURE DIRECTIONS}

In the last decade, the increasingly close MDT working between psychiatrist, neurosurgeon and neurologist combined by recognition of the limitations of current treatment options for a small but albeit significant proportion of patients with primary psychiatric disease, has refuelled interest in DBS as a therapy option once again.

The first two papers in the 'modern era of DBS in psychiatry' were published in 1999, addressing Tourette syndrome and OCD. ${ }^{35} 36$ Since then, the publication numbers have exploded and a Medline search today produced over 500 publications in relation to DBS and a range of psychiatric diseases, primarily depression, OCD and Tourette syndrome but also others including addiction and aggressive behaviour.

Interestingly, when DBS was revisited, it was mainly the original targets used for stimulation or lesioning decades before which were first re-explored rather than newer potential targets. While this is a tribute to the valuable contribution of the pioneers, it is disappointing that new targets or a firm 
pathophysiological basis for old ones has not been established despite modern neuroscience research techniques and animal models.

Tourette syndrome, a condition characterised by chronic motor and vocal tics, was the first to be treated with DBS in this modern era. In 1999, Vandewalle et $a l^{35}$ reported a 42-year-old patient with intractable Tourette syndrome who was implanted with bilateral electrodes in the original thalamic nuclei lesioned by Hassler and Dieckmann ${ }^{37}$ in three patients with Tourette syndrome in 1960s. At 1 year, the tics were reported to be abolished. Since, a number of studies, with patients implanted in the thalamic targets and/or others such as pallidum, ${ }^{38}$ have reported significant improvement in the tic scores following DBS. Reduced energy levels and change in libido are adverse events reported by some patients. The evidence, however, should be viewed critically given that it is primarily derived from case series, the largest of 18 patients, ${ }^{39}$ with randomised blinded trials being uncommon. ${ }^{40}$ The current evidence is, however, sufficient to support the development of larger randomised trials of DBS in Tourette syndrome to clarify its role. ${ }^{41}$

In 1999, Nuttin et $a l^{36}$ reported four patients with severe OCD implanted bilaterally in the anterior limb of the internal capsule, at a target traditionally used for capsulotomy in 1940s. ${ }^{42}$ They recorded significant improvement in three of them. The rationale for surgery was based on previous experience with lesioning in a host of targets, such as the internal capsule, cingulum and subcaudate tracts, in patients with severe OCD that had shown significant improvement. The territory around the anterior limb of the internal capsule, to include ventral capsule, ventral striatum and nucleus accumbens remains the commonest target for DBS in intractable OCD.

Greenberg et $a l^{43}$ recently published a review of 26 patients with severe OCD implanted in ventral capsule or ventral striatum at four different surgical centres. At 3-36 months follow-up, 62\% of the patients had at least 35\% improvement in their Yale-Brown Obsessive-Compulsive Scale (Y-BOCS) scores, meeting the full response criteria. In terms of significant adverse events, one patient had a small intracerebral haemorrhage leading to apathy which improved by 3 months and there was one electrode fracture requiring replacement.

Widespread use of DBS in patients with movement disorders, resulting in novel observations in new DBS targets, is now beginning to feed back into psychiatry. The reports of improvement in OCD traits following STN-DBS for PD ${ }^{44}$ led to a crossover, double blind, multicentre trial of STN-DBS in 16 patients with intractable OCD. ${ }^{32}$ At 3 months' follow-up, $75 \%$ of the patients had at least a $25 \%$ improvement in their Y-BOCS scores. Serious adverse events included one intracranial bleed leading to finger paralysis and two patients with infection.

Based on such evidence, in 2009 Food and Drug Administration granted a humanitarian device exemption for the use of DBS in patients with severe OCD, which was shortly followed by the receipt of CE marking in Europe. Clearly though further larger scale post-market studies are required to clearly define the optimal target, patient population and adverse event profile.

Depression is the fourth leading cause of disability worldwide and $15 \%$ of population experience at least one episode of depression in their life time. ${ }^{45}$ In the vast majority, this is selflimiting or amenable to non-interventional treatment. For a small but significant number of patients unresponsive to conventional therapy, however, surgery may have a role. Lesional surgery, including cingulotomy, leucotomy or medial thalamotomy, with its potential for high morbidity, has now given way to
DBS as the surgical treatment of choice. ${ }^{46}$ The landmark work in DBS for depression was by the psychiatrist and neurosurgeon team of Mayberg and Lozano who based on positron emission tomography studies identified subcallosal cingulate, including Brodmann area 25, as a suitable target for DBS in treatment-resistant depression. ${ }^{47}$ The group recently reported the outcome of 21 such patients implanted bilaterally in this target. At 1 year follow-up, $62 \%$ of the patients had at least a $40 \%$ reduction in the Hamilton Rating Scale for Depression scores. ${ }^{48}$ The most serious adverse event related to a patient who committed suicide early in the study. There were also two electrode fractures and one infection. Of interest, the stimulating current used in patients with treatment-resistant depression was noticeably higher than that used in movement disorder patients. One explanation could be the need to activate multiple white matter pathways to achieve remission. Another might be that the optimal target was actually away from where electrodes are currently being implanted, and therefore therapeutic effect relied on high current to spread to the 'sweet spot'.

There is presently a great deal of interest in localising the optimal target point for DBS in severe depression as well as in defining the most effective stimulating parameters. Our institution is currently a part of a multicentre European trial of DBS in intractable depression to address the latter point.

More recently, the possible utility of DBS has been investigated in a number of other psychiatric disorders. Heinze et $a l^{49}$ treated three patients with alcohol dependence with DBS of nucleus accumbens and reported patients' abstinence for significant periods of time. Laxton et $a l^{50}$ reported a phase I trial of six patients with Alzheimer's disease (AD) undergoing DBS of fornices. At 1 year follow-up, there was significant reversal of glucose metabolism in the temporal and parietal lobes of the brain. Clinically, there was a reduction in the rate of decline on mini mental state examination and indeed scores improved in two patients. There were no significant side effects. If such therapy is indeed shown to be clinically effective in $\mathrm{AD}$, then this would add further support for the potential of electrical stimulation therapy in the management of symptoms of both neurodegenerative (PD and $\mathrm{AD}$ ) and non-neurodegenerative (dystonia, OCD, Tourette syndrome and depression) disorders. Whether DBS has a role in the modification of disease progression and neuroprotection is under debate. ${ }^{51}$

\section{CONCLUSION}

DBS represents a powerful and effective technique for the treatment of a range of neurological and psychiatric conditions. As our understanding of the disease pathophysiology improves, our imaging techniques become more powerful and the technological advances allow better hardware design, it is likely that the use and indications of DBS will grow further. Despite its definite advantages over the previous lesioning and destructive techniques, DBS is by no means risk free. A multidisciplinary experienced team is an absolute requirement for delivery of this treatment. The ethical considerations also remain and particularly so when DBS surgery is applied to treat patients with psychiatric disease if the previous mistakes are to be avoided. Furthermore, we need to involve patients, carers and their support groups much more actively so as to tailor our research to their needs and meet their concerns. As we move into the future, the treatments will evolve, but the goals should remain unchanged as those of the age-old medicine: to heal and improve the life of our patients. 


\section{Main messages}

- Deep brain stimulation (DBS) is a National Institute of Clinical Excellence-approved, effective treatment for medically refractory Parkinson's disease, dystonia and tremor.

- A specialist multidisciplinary team approach is essential for patient selection and management.

- There is increasing interest in the role of DBS to treat patients with psychiatric disease. Further research, however, is required to clearly define this role.

\section{Current research questions}

- Which group of patients with psychiatric disease are likely to benefit from surgery?

- What are the optimal targets for deep brain stimulation (DBS) for the different psychiatric disorders?

- What is the patient and public perception of DBS for psychiatric disease?

\section{Key references}

- Benabid AL, Pollak P, Louveau A, et al. Combined (thalamotomy and stimulation) stereotactic surgery of the VIM thalamic nucleus for bilateral Parkinson disease. Appl Neurophysiol 1987;50:344-6.

- Williams A, Gill S, Varma T, et al. Deep brain stimulation plus best medical therapy versus best medical therapy alone for advanced Parkinson's disease (PD SURG trial): a randomised, open-label trial. Lancet Neurol 2010;9:581-91.

- Shotbolt P, Moriarty J, Costello A, et al. Relationships between deep brain stimulation and impulse control disorders in Parkinson's disease, with a literature review. Parkinsonism Relat Disord 2012;18:10-16.

- Piedad JC, Rickards HE, Cavanna AE. What patients with gilles de la tourette syndrome should be treated with deep brain stimulation and what is the best target? Neurosurgery 2012;71:173-92.

- Greenberg BD, Gabriels LA, Malone DA Jr, et al. Deep brain stimulation of the ventral internal capsule/ventral striatum for obsessive-compulsive disorder: worldwide experience. Mol Psychiatry 2010;15:64-79.

- Mayberg HS, Lozano AM, Voon V, et al. Deep brain stimulation for treatment-resistant depression. Neuron 2005;45:651-60.

Contributors This is to confirm that all authors have contributed to the paper to fulfil the criteria for authorship.

\section{Competing interests None.}

Provenance and peer review Not commissioned; externally peer reviewed.

\section{REFERENCES}

1 Delgado JM, Hamlin H, Chapman WP. Technique of intracranial electrode implacement for recording and stimulation and its possible therapeutic value in psychotic patients. Confin Neurol 1952;12:315-19.

2 Spiegel EA, Wycis HT, Marks M, et al. Stereotaxic apparatus for operations on the human brain. Science 1947;106:349-50.
3 Benabid AL, Pollak P, Louveau A, et al. Combined (thalamotomy and stimulation) stereotactic surgery of the VIM thalamic nucleus for bilateral Parkinson disease. Appl Neurophysiol 1987;50:344-6.

4 Ashkan $\mathrm{K}$, Wallace B, Bell BA, et al. Deep brain stimulation of the subthalamic nucleus in Parkinson's disease 1993-2003: where are we 10 years on? Br J Neurosurg 2004;18:19-34.

5 Samuel M, Ashkan K, Lang AE. Lesion surgeries. In: Pahwa R, Lyons K, Koller B, eds. Handbook of Parkinson's disease. 4th edn. New York: Marcel Dekker, 2007: 391-403.

6 Ashkan K, Blomstedt P, Zrinzo L, et al. Variability of the subthalamic nucleus: the case for direct MRI guided targeting. Br J Neurosurg 2007;21:197-200.

7 Deuschl G, Schade-Brittinger C, Krack P, et al. A randomized trial of deep-brain stimulation for Parkinson's disease. N Engl J Med 2006;355:896-908.

8 Weaver FM, Follett K, Stern M, et al. Bilateral deep brain stimulation vs best medical therapy for patients with advanced Parkinson disease: a randomized controlled trial. JAMA 2009;301:63-73.

9 Williams A, Gill S, Varma T, et al. Deep brain stimulation plus best medical therapy versus best medical therapy alone for advanced Parkinson's disease (PD SURG trial): a randomised, open-label trial. Lancet Neurol 2010;9:581-91.

10 Eltahawy HA, Saint-Cyr J, Giladi N, et al. Primary dystonia is more responsive than secondary dystonia to pallidal interventions: outcome after pallidotomy or pallidal deep brain stimulation. Neurosurgery 2004;54:613-19.

11 Pahwa R, Lyons KE, Wilkinson SB, et al. Comparison of thalamotomy to deep brain stimulation of the thalamus in essential tremor. Mov Disord 2001;16:140-3.

12 The National Institute for Health and Clinical Excellence. Deep brain stimulation for Parkinson's disease. http://www.nice.org.uk/guidance//PG19 (accessed 15 May 2012).

13 The National Institute for Health and Clinical Excellence. Deep brain stimulation for tremor and dystonia (excluding Parkinson's disease). http://www.nice.org.uk/ guidance//PG188 (accessed 15 May 2012).

14 Bergman H, Wichmann T, Karmon B, et al. The primate subthalamic nucleus. II. Neuronal activity in the MPTP model of parkinsonism. J Neurophys 1994;72:507-20.

15 Alexander GE, Delong MR, Strick PL. Parallel organization of functionally segregated circuits linking basal ganglia and cortex. Annu Rev Neurosci 1986;9:357-81.

16 Hammond C, Bergman H, Brown P. Pathological synchronization in Parkinson's disease: networks, models and treatments. Trends Neurosci 2007;30:357-64.

17 Lang AE, Houeto IL, Krack P, et al. Deep brain stimulation: Preoperative issues. Mov Disord 2006;21(Suppl 14):S171-96.

18 Houeto JL, Mesnage V, Mallet L, et al. Behavioural disorders, Parkinson's disease and subthalamic stimulation. J Neurol Neurosurg Psychiatry 2002;72:701-7.

19 Schüpbach M, Gargiulo M, Welter ML, et al. Neurosurgery in Parkinson disease: a distressed mind in a repaired body? Neurology 2006;66:1811-16.

20 Saint-Cyr JA, Trepanier LL, Kumar R, et al. Neuropsychological consequences of chronic bilateral stimulation of the subthalamic nucleus in Parkinson's disease. Brain 2000:123:2091-108.

21 Voon V, Saint-Cyr J, Lozano AM, et al. Psychiatric symptoms in patients with Parkinson disease presenting for deep brain stimulation surgery. J Neurosurg 2005; 103:246-51.

22 Beck AT, Ward CH, Mendelson $\mathrm{M}$, et al. An inventory for measuring depression. Arch Gen Psychiatry 1961;4:561-71.

23 Zigmond AS, Snaith RP. The hospital anxiety and depression scale. Acta Psychiatrica Scandinavica 1983:67:361-70.

24 Smeding HM, Goudriaan AE, Foncke EM, et al. Pathological gambling after bilateral subthalamic nucleus stimulation in Parkinson disease. J Neurol Neurosurg Psychiatry 2007;78:517-19

25 Romito LM, Raja M, Daniele A, et al. Transient mania with hypersexuality after surgery for high frequency stimulation of the subthalamic nucleus in Parkinson's disease. Mov Disord 2002;17:1371-4.

26 Ardouin C, Voon V, Worbe Y, et al. Pathological gambling in Parkinson's disease improves on chronic subthalamic nucleus stimulation. Mov Disord 2006;21:1941-6.

27 Bandini F, Primavera A, Pizzorno M, et al. Using STN DBS and medication reduction as a strategy to treat pathological gambling in Parkinson's disease. Parkinsonism Relat Disord 2007:13:369-71.

28 Shotbolt P, Moriarty J, Costello A, et al. Relationships between deep brain stimulation and impulse control disorders in Parkinson's disease, with a literature review. Parkinsonism Relat Disord 2012;18:10-16

29 Alegret $\mathrm{M}$, Junqué $\mathrm{C}$, Valldeoriola $\mathrm{F}$, et al. Obsessive-compulsive symptoms in Parkinson's disease. J Neurol Neurosurg Psychiatry 2001;70:394-6.

30 Mallet L, Mesnage V, Houeto JL, et al. Compulsions, Parkinson's disease, and stimulation. Lancet 2002;360:1302-4.

31 Fontaine $\mathrm{D}$, Mattei $\mathrm{V}$, Borg $\mathrm{M}$, et al. Effect of subthalamic nucleus stimulation on obsessive compulsive disorder in a patient with Parkinson disease. Case report. J Neurosurg 2004;100:1084-6.

32 Mallet L, Polosan M, Jaafari N, et al. Subthalamic nucleus stimulation in severe obsessive-compulsive disorder. N Engl J Med 2008;359:2121-34.

33 Mioshi E, Dawson K, Mitchell J, et al. The Addenbrooke's Cognitive Examination Revised (ACE-R): a brief cognitive test battery for dementia screening. Int I Geriatr Psychiatry 2006;21:1078-85. 
34 Mental Capacity Act. London: HMSO, 2005

35 Vandewalle V, van der Linden C, Groenewegen HJ, et al. Stereotactic treatment of Gilles de la Tourette syndrome by high frequency stimulation of thalamus. Lancet 1999;353:724.

36 Nuttin B, Cosyns P, Demeulemeester $\mathrm{H}$, et al. Electrical stimulation in anterior limbs of internal capsules in patients with obsessive-compulsive disorder. Lancet 1999:354:1526.

37 Hassler R, Dieckmann G. Stereotaxic treatment of tics and inarticulate cries or coprolalia considered as motor obsessional phenomena in Gilles de la Tourette's disease. Rev Neurol (Paris) 1970;123:89-100.

38 Houeto JL, Karachi C, Mallet L, et al. Tourette's syndrome and deep brain stimulation. J Neurol Neurosurg Psychiatry 2005;76:992-5.

39 Servello D, Porta M, Sassi M, et al. Deep brain stimulation in 18 patients with severe Gilles de la Tourette syndrome refractory to treatment: the surgery and stimulation. J Neurol Neurosurg Psychiatry 2008;79:136-42.

40 Welter ML, Mallet L, Houeto $\mathrm{J}$, et al. Internal pallidal and thalamic stimulation in patients with Tourette syndrome. Arch Neurol 2008;65:952-7.

41 Piedad JC, Rickards HE, Cavanna AE. What patients with gilles de la tourette syndrome should be treated with deep brain stimulation and what is the best target? Neurosurgery 2012;71:173-92.

42 Bingley T, Leksell L, Meyerson BA, et al. Long-term results of stereotactic anterior capsulotomy in chronic obsessive-compulsive neurosis. In: Sweet WH, Obrador S, Martín-Rodríguez JG, eds. Neurosurgical treatment in psychiatry, pain, and epilepsy. Baltimore: University Park Press, 1977: 287-99.
43 Greenberg BD, Gabriels LA, Malone DA Jr, et al. Deep brain stimulation of the ventral internal capsule/ventral striatum for obsessive-compulsive disorder: worldwide experience. Mol Psychiatry 2010;15:64-79.

44 Alegret $\mathrm{M}$, Junqué $\mathrm{C}$, Valldeoriola $\mathrm{F}$, et al. Effects of bilateral subthalamic stimulation on cognitive function in Parkinson disease. Arch Neurol 2001;58:1223-7.

45 The National Health Service Evidence. Depression - How common is it? http://www. cks.nhs.uk/depression/background_information/prevalence (accessed 15 May 2012).

46 Bjarkam CR, Sorensen JC. Psychosurgery—a historical perspective. In: Lozano AM, Gildenberg PL, Tasker RR, eds. Textbook of stereotactic and functional neurosurgery. 2nd edn. Berlin: Springer, 2009: 2867-86.

47 Mayberg HS, Lozano AM, Voon V, et al. Deep brain stimulation for treatmentresistant depression. Neuron 2005;45:651-60.

48 Lozano AM, Giacobbe P, Hamani C, et al. A multicenter pilot study of subcallosal cingulate area deep brain stimulation for treatment-resistant depression. J Neurosurg 2012;116:315-22.

49 Heinze HJ, Heldmann M, Voges J, et al. Counteracting incentive sensitization in severe alcohol dependence using deep brain stimulation of the nucleus accumbens: clinical and basic science aspects. Front Hum Neurosci 2009:3:22.

50 Laxton AW, Tang-Wai DF, McAndrews MP, et al. A phase I trial of deep brain stimulation of memory circuits in Alzheimer's disease. Ann Neurol 2010;68:521-34.

51 Wallace $B A$, Ashkan $K$, Heise $C E$, et al. Survival of midbrain dopaminergic cells after lesion or deep brain stimulation of the subthalamic nucleus in MPTP-treated monkeys. Brain 2007;130:2129-45. 